\title{
Marcus Twellmann
}

\section{Chamambo: Dorfgeschichten im globalen Vergleich}

\begin{abstract}
Despite its cultural specificity the concept of, village stories' can be used for comparative literary studies with a global scope. Taking Berthold Auerbach's early Schwarzwälder Dorfgeschichten (1843) as a starting point the following article puts this claim to the test in reading Gabriel Ruhumbika's Village in Uhuru (1969), an English-language novel from Tanzania, as an exemplar of the same genre.
\end{abstract}

DOI 10.1515/iasl-2015-0004

1

Seit den frühen 1840er Jahren bezeichnet das Wort ,Dorfgeschichte‘ im deutschen Sprachraum eine literarische Form. Zwar wurde vorher schon vom Dorf erzählt, doch haben Berthold Auerbachs Schwarzwälder Dorfgeschichten die Gattung erst eigentlich begründet. Es handelt sich dabei allerdings um ein gesamteuropäisches Phänomen: In England, Frankreich, Spanien, Ungarn, Schweden, Holland, Dänemark, Russland und anderen Ländern Europas erscheinen in den 1840er Jahren vermehrt literarische Erzählungen vom ländlichen und zumeist dörflichen Leben. ${ }^{1}$ Dass Auerbach dabei eine besondere Bedeutung zukommt, hat Ivan Turgenev 1868 bemerkt:

Unvergeßlich bleibt der Eindruck, welchen das Erscheinen der ,Schwarzwälder Dorfgeschichten' in der ersten Hälfte der vierziger Jahre in Deutschland hervorrief. Die Hinneigung der gesammten Europäischen Litteratur zum Volksleben ist um dieselbe Zeit unverkennbar [...] - doch die Ehre der Initiative, des ersten Anfangs bleibt bei Auerbach. ${ }^{2}$

1 Siehe dazu Rudolf Zellweger: Les débuts du roman rustique. Suisse, Allemagne, France, 1836-1856. Paris: Droz 1941.

2 Ivan S. Turgenev: Eine unbekannte deutsche Handschrift von Turgenevs Auerbach-Vorrede. In: Gerhard Ziegengeist (Hg.): I.S. Turgenev und Deutschland. Materialien und Untersuchungen. Berlin: Akademie Verlag 1965, S. 68-75, hier S. 70.

PD Dr. Marcus Twellmann: Forschungsstelle Kulturtheorie, Universität Konstanz, Fach 213, 78457 Konstanz, E-Mail: marcus.twellmann@uni-konstanz.de 
Die Erzählungen aus Schwaben wurden weltweit gelesen. In englischen Zeitschriften etwa ist Auerbach zwischen 1841 und 1850 einer der drei meistgenannten deutschsprachigen Schriftsteller dieser Zeit. Auch außerhalb von Europa fanden seine Erzählungen Verbreitung. Für das 19. Jahrhundert wurden 240 Übertragungen in 18 Sprachen gezählt. ${ }^{3}$

Seither haben Schriftsteller in vielen Teilen der Welt Geschichten vom Dorf verfasst und tun es noch heute. Vermutlich sind den meisten Autoren Auerbachs Texte gänzlich unbekannt und sicher würden die wenigsten ihre Erzählungen selbst einer Gattung namens ,Dorfgeschichte' zuordnen, von der sich bisher vornehmlich deutsche Literaten und Literaturwissenschaftler einen bestimmten Begriff gemacht haben. Will man gleichwohl Geschichten aus aller Welt zusammenziehen und auf generische Regelmäßigkeiten hin untersuchen, dann kann ein solches Unternehmen sich in der Mehrzahl der Fälle also nicht auf ein emisches, mit der literarischen Produktion und Rezeption durchgehend verbundenes und in den Quellen nachweisbares Konzept oder gar auf dort auffindbare Poetologien der Dorfgeschichte stützen. Zumeist lässt die Gattung sich nur mit einer etischen Perspektive von außen betrachten. Das emische Gattungskonzept des 19. Jahrhunderts soll im Folgenden indes als ein Klassifikationsbegriff genutzt werden, um transnational und -kontinental, wenn nicht ,transkulturell' wiederkehrende Merkmale von Texten zu erfassen, die in keinem leicht nachweisbaren Traditionszusammenhang stehen. Darum kann ihre Vergleichbarkeit nicht als gegeben vorausgesetzt werden, sie ist allererst herzustellen. Auf diese Weise könnten Autoren aus großer Entfernung ins Gespräch gebracht werden, die voneinander nicht wussten, und doch eine geteilte Geschichte erzählten.

Dieses Vorhaben begegnet einer Schwierigkeit wieder, mit der sich schon die ältere Germanistik konfrontiert sah: Da sich distinktive Merkmale formaler Art kaum angeben ließen, konnte die Gattungsdefinition nur beim Stoff ansetzen. So heißt es schon in der ersten systematisch angelegten Monographie zu diesem Thema: „Die Dorfgeschichte spielt im Dorf und handelt von Bauern. Dies ist die einzige Feststellung, die wir für die gesamte Dorfepik machen können.“4 Sie gilt nach wie vor. Die bisher aufschlussreichste Studie zu den deutschsprachigen Texten hat sich der Gattung sozialgeschichtlich genähert und nach ihrer gesellschaftlichen Funktion gefragt. ${ }^{5}$ Will man diese Herangehensweise über den nationalen Rahmen hinaus mit einer globalen Perspektive verfolgen, sind zunächst

3 Angaben nach Uwe Baur: Dorfgeschichte. Zur Entstehung und gesellschaftlichen Funktion einer literarischen Gattung im Vormärz. München: Fink 1978, S. 237 f.

4 Friedrich Altvater: Wesen und Form der deutschen Dorfgeschichte im 19. Jahrhundert. Berlin: Kraus 1930, S. 13.

5 Baur: Dorfgeschichte (Anm. 3). 
weitere Unschärfen in Kauf zu nehmen. Denn was ,Dörfer ${ }^{66}$ sind und was ,Bauern', ${ }^{7}$ ist unter Historikern, Anthropologen und Geographen durchaus nicht unumstritten. Diese definitorischen Unbestimmtheiten machen es indes möglich, im Rahmen einer konstruktiven Komparatistik unterschiedlichste literarische Artefakte als Dorfgeschichten zu betrachten. Ein genauerer Gattungsbegriff ist nicht im Vorhinein zu bilden, er könnte allenfalls das Ergebnis solcher Betrachtungen sein. Zunächst gilt es Marcel Detiennes Anleitung zu historisch-anthropologischen Vergleichsstudien folgend „Komparablen“ zu konstruieren. ${ }^{8}$

Ob global vergleichende Untersuchungen möglich und heuristisch ertragreich sind, soll hier an einem Text erprobt werden, der von Auerbachs Erzählungen in verschiedener Hinsicht weit entfernt ist: Village in Uhuru, ${ }^{9}$ ein tansanischer Roman, den der 1938 als ein Mitglied der Herrscherfamilie von Ukerewe, einer Insel im Viktoriasee, geborene Gabriel Ruhumbika ${ }^{10}$ in englischer Sprache verfasst hat. 1969 ist der Text in der Reihe African Creative Writing bei Longman in London erschienen. Auf den ersten Blick scheint ihn nichts mit den Schwarzwälder Dorfgeschichten zu verbinden. Eben deshalb könnte ein Vergleich aber aufschlussreich sein. Denn die Ertragsaussichten eines solchen Unternehmens vergrößern sich mit der Entfernung zwischen den Vergleichsgegenständen. Das gilt freilich nur dann, wenn die Untersuchung des tansanischen Romans sich nicht darin erschöpft, einen Merkmalskatalog abzufragen, der zuvor auf Grundlage der deutschsprachigen Texte erstellt wurde. Vielmehr ist der Vergleich so anzulegen, dass auch der bereits sehr viel besser erforschte und darum vielleicht allzu vertraute Gegenstand sich von dem neu herangezogenen her in einer veränderten Perspektive zeigen kann. Wenn sich also mögliche Vergleichsgesichtspunkte auch zunächst nur ausgehend vom germanistischen Forschungsstand vorzeichnen lassen, so sind diese gleichwohl als veränderliche $\mathrm{zu}$ handhaben, so dass sich in der Befassung mit der Dorfgeschichte Ruhumbikas die Auerbach'schen vielleicht neu erschließen.

Für die frühen Dorfgeschichten konnte in historischer Hinsicht gezeigt werden, dass sie in Auseinandersetzung mit den Prozessen der Staats- und Nations-

6 Siehe z. B. Jonathan Rigg: Redefining the Village and Rural Life: Lessons from South East Asia. In: Geographic Journal 160 (1994), S. 123-135.

7 Siehe z.B. Michael Kearney: Reconceptualizing the Peasantry: Anthropology in Global Perspective. Boulder: Westview 1996.

8 Siehe dazu Marcel Detienne: Comparer l'incomparable. Paris: Seuil 2000, S. 41-80.

9 Gabriel Ruhumbika: Village in Uhuru. London: Longman 1969. Zitate daraus werden im Text mit der Sigle VU und Seitenangabe nachgewiesen.

$10 \mathrm{Vgl}$. Douglas Killam: Literature of Africa: Literature as Windows to World Cultures. Westport: Greenwood Press 2004, S. 181. 
bildung entstanden sind. ${ }^{11}$ Gemeinsam ist ihnen ein enger Ortsbezug: Auerbachs Erzählungen handeln von seinem Heimatdorf Nordstetten bei Horb am Neckar in Württemberg. Das seit 1806 souveräne Königreich betrieb die innere Staatsbildung in der ersten Hälfte des 19. Jahrhunderts mit Nachdruck. Im Zuge der administrativen Integration griff der Staat mit neuartiger Intensität auf die lokale Ebene durch. Da man sich im südwestdeutschen Raum mit besonderem Nachdruck für eine kommunale Selbstverwaltung einsetzte, führte diese Regierungsintensivierung einen Konflikt herauf, der sich in den unruhigen Jahren vor 1850 zuspitzte. Dieser Konflikt zwischen den Autonomieansprüchen einer dörflichen Landgemeinde und den Hoheitsansprüchen des sich formierenden Staats wird in Auerbachs Erzählungen thematisch und prägt zudem seine Poetik einer volkstümlichen Literatur, die er mit einem Seitenblick auf bürokratische Schriftpraktiken entwickelt.

Ist Village in Uhuru in einem vergleichbaren Umfeld gesellschaftlicher Entwicklungen entstanden? Die Frage ist insofern zumindest nicht unangebracht, als es sich dabei um einen historischen Roman handelt, der auf reale Personen, Orte und Ereignisse der Zeitgeschichte Bezug nimmt. Das Wort „Uhuru“, Swahili für ,Freiheit‘, bedeutet hier vor allem ,politische Unabhängigkeit‘. Schon der Titel ist auf die Geschichte Tansanias zu beziehen: Nachdem die seit 1885 bestehende Kolonie Deutsch-Ostafrika erobert worden war, hatte das Tanganyika Territory ab 1918 unter britischer Herrschaft gestanden. Die Romanhandlung setzt in den 1940er Jahren ein und reicht über die Loslösung Tanganyikas vom Vereinigten Königreich am 9. Dezember 1961 und die Gründung des Staates Tansania bis in die zweite Hälfte der 1960er Jahre. Über das, was im Titel „village“ heißt, wird der mit europäischen Dorfgeschichten vertraute Leser erfahren, dass es in diesem Umfeld eine ungewohnte Bedeutung hat: Die gesellschaftliche Neuordnung geht nicht mit der Auflösung des Dorfes einher, sondern mit seiner Entstehung.

Der tansanische Roman handelt von Vorgängen, die dem 19. Jahrhundert weitgehend unbekannt waren. In Russland hatten sich nach der Oktoberrevolution 1917 erste kollektiv bewirtschaftete Agrarbetriebe gebildet. Neben diesen ,Kolchosen" wurden bald auch ,Sowchosen', staatliche Landwirtschaftsgroßbetriebe, eingerichtet. Ab 1928 trieb man den Prozess der Kollektivierung auch unter Anwendung von Zwang voran. ${ }^{12}$ Nachdem das Land im Zuge einer 1949 begonne-

11 Siehe dazu Marcus Twellmann: Literatur und Bürokratie im Vormärz. Zu Berthold Auerbachs Dorfgeschichten. In: Deutsche Vierteljahrsschrift für Literaturwissenschaft und Geistesgeschichte (DVjs) 86/4 (2012), S. 578-608.

12 Siehe dazu Stephan Merl: Die Anfänge der Kollektivierung in der Sowjetunion. Der Übergang zur staatlichen Reglementierung der Produktions- und Marktbeziehungen im Dorf (1928-1930). Wiesbaden: Harrassowitz 1985; S.M.: Bauern unter Stalin. Die Formierung des sowjetischen Kolchossystems 1930-1941. Berlin: Duncker \& Humblot 1990; Lynne Viola: Peasant Rebels under 
nen Bodenreform neu verteilt worden war, ging man auch in China 1952 zur Kollektivierung über. Volkskommunen wurden eingerichtet, in denen jeweils etwa 5.000 bäuerliche Haushalte zusammengeschlossen waren. ${ }^{13}$ In beiden Fällen führte die wirtschaftliche Neuordnung zu einer tiefgreifenden Veränderung älterer dörflicher Strukturen. In Tansania dagegen ging die Kollektivierung der Agrarproduktion mit der Neubildung von Dörfern einher. „Village“ heißt in Ruhumbikas Roman eine von tausenden ländlicher Siedlungen, die im Zuge staatlich gesteuerter Villagization entstanden.

Wenn die gesellschaftshistorischen Voraussetzungen der Dorfgeschichten sich auch erheblich unterscheiden, so fällt eine Gemeinsamkeit doch ins Auge: Sie handeln in jedem Fall von einer Veränderung lokaler Verhältnisse unter der Einwirkung translokaler Prozesse. Darauf kann die folgende Arbeitsdefinition sich stützen: Die Dorfgeschichte ist eine literarische Form, in der sich Erfahrungen mit Transformationsprozessen artikulieren. Sie stellt die Veränderung ländlicher Lebensformen im Zuge translokaler Prozesse aus lokaler Perspektive dar. ${ }^{14}$ Die hohe Abstraktionslage dieser vorläufigen Gattungsbestimmung trägt dem Umstand Rechnung, dass auch im vermeintlichen ,Zeitalter der Nationalstaaten“ imperiale Formationen noch vorherrschend waren. ${ }^{15}$ Mit einer solchen hatten die Verfasser russischer Dorfgeschichten des 19. Jahrhunderts, so etwa Nikolai Leskov zu tun wie auch jene, die nach ihnen die Sowjetisierung der Landwirtschaft literarisch begleiteten, z.B. Andrej Platonov, Michail Šolochov und Fedor Panferov. Das gilt weiterhin für chinesische Autoren wie Zhou Libo oder Ding Ling und auch für die Inder Raja Rao und Premtschand. Ruhumbikas Roman aber ist nach der Dekolonisierung Ostafrikas in einem gesellschaftlichen Umfeld entstanden, das von Staats- und Nationsbildungsanstrengungen geprägt war. „Every man woman and child was burning with the fire of nationalism“ (VU, S. 66), heißt es im Text. Retrospektiv lässt sich feststellen, dass diese Begeisterung vor allem zentralisierte Strukturen staatlicher Verwaltung gestärkt hat:

Stalin: Collectivization and the Culture of Peasant Resistance. Oxford: Oxford University Press 1996.

13 Siehe dazu Dali L. Yang: Calamity and Reform in China: State, Rural Society, and Institutional Change since the Great Leap Famine. Stanford: Stanford University Press 1996 sowie Edward Friedman / Paul G. Pickowicz / Mark Selden: Revolution, Resistance, and Reform in Village China. New Haven: Yale University Press 2005.

14 Siehe dazu Michael Neumann / Marcus Twellmann: Dorfgeschichten. Anthropologie und Weltliteratur. In: Deutsche Vierteljahrsschrift für Literaturwissenschaft und Geistesgeschichte (DVjs) 88/1 (2014), S. 22-45.

15 Vgl. Jürgen Osterhammel: Die Verwandlung der Welt. Eine Geschichte des 19. Jahrhunderts. München: Beck 2009, S. 583f. 
Nation-building ideologies [...] as it turned out were state-building ideologies in which the State itself was modelled on the colonial despotic State. Concentration of power in the executive arm of the State - whether this came about through military interventions or oneparty populism of the right or the left - was the defining characteristic of the post-independence African State. ${ }^{16}$

Village in Uhuru aber wurde wenige Jahre nach der Gründung eines unabhängigen tansanischen Staats geschrieben, zu einer Zeit, als diese Charakteristika noch weniger deutlich hervortraten.

Weltweit, eine Verflechtungsgeschichte der Literatur muss das berücksichtigen, ${ }^{17}$ haben Gesellschaften das europäische Modell importiert, so auch viele postkoloniale Gesellschaften des 20. Jahrhunderts. ${ }^{18}$ Die globale Ausbreitung von Nationalstaatlichkeit ist Teil der Verwestlichung der Welt, zugleich hat sie das System der großen westlichen Übersee-Imperien unterwandert. ${ }^{19}$ Der Nationalismus war in den Kolonien zunächst eine antikoloniale Ideologie indigener Eliten, die politische Selbständigkeit anstrebten. Nach der Unabhängigkeit wurde er zu einem Mittel der Staatsbildung, von der man sich eine umfassende Modernisierung und Verbesserung der Lebensbedingungen versprach. Erforderlich war es zu diesem Zweck nicht zuletzt, „to ,construct a national culture““. ${ }^{20}$ Ruhumbika hat sich zu diesem Projekt 1974 geäußert und sich dabei auf Julius K. Nyerere bezogen, den Führer der Tanganyika African National Union (TANU) und ersten Staatspräsidenten des Landes, sowie auf Frantz Fanon, ${ }^{21}$ dessen Les damnés de la

16 Issa G. Shivij: Critical elements of a new democratic consensus in Africa. In: I.G.S.: Where Is Uhuru? Reflections on the Struggle for Democracy in Africa. Hg. von Godwin R. Murunga. Cape Town u. a.: Fahamu 2009, S. 8-19, hier S. 13.

17 Siehe dazu Annette Werberger: Überlegungen zu einer Literaturgeschichte als Verflechtungsgeschichte. In: Dorothee Kimmich / Schamma Schahadat (Hg.): Kulturen in Bewegung. Beiträge zur Theorie und Praxis der Transkulturalität. Bielefeld: Transcript 2012, S. 109-141.

18 Siehe dazu Wolfgang Reinhard: Geschichte der Staatsgewalt und europäische Expansion. In: W.R. (Hg.): Verstaatlichung der Welt? Europäische Staatsmodelle und außereuropäische Machtprozesse. München: Oldenbourg 1999, S. 317-356.

19 Vgl. Andreas Eckert: Anti-Western Doctrines of Nationalism. In: John Breuilly (Hg.): The Oxford Handbook of the History of Nationalism. Oxford: Oxford University Press 2013, S. 56-74, hier S. 59.

20 Louis A. Mbughuni / Gabriel Ruhumbika: TANU and National Culture. In: G.R. (Hg.): Towards Ujamaa: Twenty Years of TANU Leadership. Nairobi: East African Literature Bureau 1974, S. 275-287, hier S. 275.

21 Zur Bedeutung der Schriften Frantz Fanons für das Selbstverständnis afrikanischer Romanschriftsteller siehe M. Keith Booker: Writing for the Wretched of the Earth: Frantz Fanon and the Radical African Novel. In: M.K.B. / Dubravka Juraga (Hg.): Rereading Global Socialist Cultures after the Cold War: The Reassessment of a Tradition. New York: Praeger 2002, S. 115-148, hier S. 115-117. 
terre Ruhumbika später gemeinsam mit Clement Maganga auch übersetzen sollte. ${ }^{22}$ Nyerere hatte Kultur als „the essence and spirit of any nation“23 definiert, Fanon als „the whole body of efforts made by a people in the sphere of thought to describe, justify and praise the action through which that people created itself and keeps itself in existence“. ${ }^{24}$ Wie Ruhumbika mit Verweis auf Leo Trotzkijs Kritik an den „Bauerntümlern“25 betont, bestand die Aufgabe nicht etwa darin, eine nationale Kultur durch die Rückwendung auf ,tribal customs and traditions“ ${ }^{26}$ wiederzugewinnen, deren Reste im Leben der Landbevölkerung die Kolonialherrschaft überdauert hätten: „We believe that the indigenous culture of the peoples of Tanzania has meaning only if it has a place in the Tanzanian reality of today and can help the construction of the Tanzania of tomorrow. “27

Damit ist ein Verhältnis von National- und Stammeskultur angesprochen, das Ruhumbika zuvor literarisch behandelt hatte. Wie Village in Uhuru vor Augen führt, waren die Sitten und Gebräuche der unterschiedlichen Stammesverbände nicht nur von untergeordneter Bedeutung, sie stellten geradezu ein Hemmnis dar für die Einigung Tansanias. Die tribale Identität stand einer nationalen Identifizierung im Wege. Dabei waren jene vermeintlich ethnisch geprägten Gruppen weniger ein Überbleibsel aus vorkolonialer Zeit als vielmehr, so hat man zugespitzt formuliert, eine ,Erfindung ${ }^{28}$ von Missionaren und Kolonialbeamten, die jene ,Stämme' für administrative Zwecke allererst konstruierten, denen sie die indigene Bevölkerung zuordneten. „Europeans believed Africans belonged to tribes; Africans built tribes to belong to“, ${ }^{29}$ schreibt John Iliffe in seiner Geschichte Tanganyikas. Jedenfalls wurden afrikanische Traditionen von den Europäern neu interpretiert und vorfindliche Gruppierungen den Anforderungen der Verwaltung

22 Frantz Fanon: Viumbe Waliolaaniwa. Dar es Salaam: Tanzania Publishing House 1978.

23 Julius K. Nyerere: President's Inaugural Address. In: J.K.N.: Freedom and Unity. Uhuru na Umoja. A Selection from Writings and Speeches 1952-65. London u. a.: Oxford University Press, S. 176-187, hier S. 186.

24 Mbughuni / Ruhumbika: TANU and National Culture (Anm. 20), S. 275. Vgl. Frantz Fanon: Die Verdammten dieser Erde. Frankfurt/M.: Suhrkamp 1981, S. 198.

25 Vgl. Leo Trotzkij: Literatur und Revolution. Nach der russischen Erstausgabe übers. von Eugen Schäfer und Hans von Riesen. Berlin: Gehrhardt 1968, S. 78-90.

26 Mbughuni / Ruhumbika: TANU and National Culture (Anm. 20), S. 276.

27 Mbughuni / Ruhumbika: TANU and National Culture (Anm. 20), S. 277. Siehe in diesem Zusammenhang Kelly M. Askew: Performing the Nation. Swahili Music and Cultural Politics in Tanzania. Chicago: University of Chicago Press 2002.

28 So im Anschluss an Eric J. Hobsbawm / Terence Ranger (Hg.): The Invention of Tradition. Cambridge: Cambridge University Press 1993.

29 John Iliffe: A Modern History of Tanganyika. Cambridge: Cambridge University Press 1979, S. 324. 
entsprechend verändert. Leitend war dabei die Vorstellung des ,Stammes` als einer Sprach-, Kultur-, Abstammungs- und politischen Gemeinschaft mit abgegrenztem Territorium. ${ }^{30}$

Diese wirkmächtige ,Imagination ${ }^{631}$ hatte reale Auswirkungen, denen die Regierungen der nachkolonialen Staaten Afrikas Rechnung zu tragen hatten. Eine Besonderheit der afrikanischen Geschichte ist der tansanische Versuch, ethnische Gruppen durch einen Sprachnationalismus zu integrieren. Während die große Mehrheit der afrikanischen Staaten an den europäischen Kolonialsprachen festhielt, wurde in Tansania Swahili zur Nationalsprache erklärt und als Verkehrssprache in fast allen Bereichen des öffentlichen Lebens durchgesetzt. Die deutschen und englischen Kolonialverwaltungen hatten mit dieser Sprachpolitik begonnen und damit der nationalen Integration vorgearbeitet. „The country was exceptionally fortunate“, bemerkt Ruhumbika, „in that by the time it got to independence its numerous sub-cultures which identify its numerous tribes were already in the process of being swallowed by a new and homogenious [sic] culture identified by the whole country’s use of Kiswahili.“32

Eine besondere Rolle spielten bei dem Aufbau einer nationalen Kultur jene Verwaltungs- und Bildungseinrichtungen, die im Kolonialzeitalter von Europäern geschaffen worden waren. Diese brachten nicht nur eine Idee der Nation, ein Staatsmodell, Organisationsformen und Verwaltungstechniken nach Afrika, sondern auch eine Literatur, die sich mit indigenen Praktiken des Schriftgebrauchs verband. Bis in die Antike, so wird vermutet, könnte man eine Swahili-Literatur wohl zurückverfolgen. Erhalten sind Manuskripte von Versdichtungen aus dem 18. Jahrhundert; ${ }^{33}$ das älteste zurzeit bekannte Epos stammt aus dem Jahr $1728 .{ }^{34}$ Erste Prosaformen sind im Bereich höfischer Geschichtschronik nachweisbar. An diese Tradition anschließend verfasste James Mbotela Uhuru wa Watumwa (Die Befreiung der Sklaven), eine 1934 erschienene, halb historische Erzählung, die als

30 Vgl. Andreas Eckert: Nation, Staat und Ethnizität in Afrika im 20. Jahrhundert. In: Arno Sonderegger / Ingeborg Grau / Birgit Englert (Hg.): Afrika im 20. Jahrhundert. Geschichte und Gesellschaft. Wien: Promedia 2011, S. 40-59, hier S. 45.

31 Terence Ranger: The Invention of Tradition Revisited: The Case of Colonial Africa. In: T.R. / Olufemi Vaughan (Hg.): Legitimacy and the State in Twentieth-Century Africa. Basingstoke: MacMillan 1993, S. 62-111.

32 Mbughuni / Ruhumbika: TANU and National Culture (Anm. 20), S. 277.

33 Vgl. Alamin Mazrui: Swahili Beyond the Boundaries: Literature, language and identity. Athens: Ohio University Press 2007, S. 16.

34 Vgl. Thomas Geider: Die Ökumene des swahili-sprachigen Ostafrika. In: Özkan Ezli / Dorothee Kimmich / Annette Werberger (Hg.): Wider den Kulturenzwang - Migration, Kulturalisierung und Weltliteratur. Bielefeld: Transcript 2009, S. 361-402, hier S. 372. 
Vorläufer des Swahili-Romans gilt. ${ }^{35}$ Zuvor hatten christliche Missionare nicht nur Teile der Bibel, sondern auch literarische Texte wie Charles Lambs Tales from Shakespeare, John Bunyans Pilgrim's Progress und Aesops Fabeln in Swahili übersetzt. ${ }^{36}$ Darüber, dass die Form des Romans in der Swahili-Literatur auf europäische Einflüsse zurückzuführen ist, herrscht weitgehend Einigkeit. ${ }^{37}$

Mit der Schriftsprache vertraut war zunächst jedoch nur eine kleine Elite, die im kolonialen Schulsystem eine Ausbildung erhielt. Hier wurden Afrikanern auch ästhetische Konventionen und Formen der europäischen Literatur vermittelt. Für Unterrichtszwecke wurden Klassiker der englischen Literatur, u.a. Stevensons Treasure Island, Swifts Gulliver's Travels und Carrolls Alice in Wonderland, übertragen. ${ }^{38}$ Die Bevölkerung der Kolonien wurde so auch zu einer eigenen Literaturproduktion angeregt. Ein 1948 gegründetes East African Literature Bureau sollte sie dabei unterstützen. Mit europäischer Literatur kamen tansanische Intellektuelle überdies im Rahmen von Studienaufenthalten im Ausland in Berührung. Nachdem er das Makerere College in Uganda - bis zur Unabhängigkeit die einzige universitäre Einrichtung Ostafrikas - besucht hatte, ${ }^{39}$ studierte Ruhumbika an der Pariser Sorbonne. Nyerere, der am Makerere College zum Lehrer ausgebildet worden war, ${ }^{40}$ studierte von 1949 bis 1952 als erster Tanganjikaner in Großbritannien an der Universität Edinburgh und übersetzte später Dramen von Shakespeare, u. a. Julius Caesar $(1963,1969)$ in die Sprache seines Landes.

Auf die Bedeutung literarisierter Bildungseliten in Prozessen der Nationsbildung ist vielfach hingewiesen worden. ${ }^{41}$ Nach der Unabhängigkeit kam auch im vorliegenden Zusammenhang den Schriftstellern eine besondere Funktion zu. In vieler Hinsicht ist sie jener der europäischen Literaten des 18. und 19. Jahrhunderts vergleichbar. Doch sollte der Prozess der Staats- und Nationsbildung sich im postkolonialen Afrika unter deutlich anderen Ausgangsbedingungen vollziehen. Weder war eine Infrastruktur der Buchproduktion und -distribution gegeben, noch gab es ein großes literarisiertes Publikum, aus dem sich eine ,Nation“ hätte bilden können. Nicht zuletzt mangelte es Tansania, wo noch heute mehr als 120 verschiedene Sprachen in Gebrauch sind, an linguistischer Einheit. Den

35 Vgl. Mazrui: Swahili Beyond the Boundaries (Anm. 33), S. 26.

36 Vgl. Mazrui: Swahili Beyond the Boundaries (Anm. 33), S. 124 f. Siehe dazu auch Jack D. Rollins: A History of Swahili Prose. Leiden: Brill 1983, S. 62.

37 Vgl. Mazrui: Swahili Beyond the Boundaries (Anm. 33), S. 175.

38 Vgl. Mazrui: Swahili Beyond the Boundaries (Anm. 33), S. 25.

39 Vgl. Killam: Literature of Africa (Anm. 10), S. 182.

40 Vgl. Andreas Eckert: Herrschen und Verwalten. Afrikanische Bürokraten, staatliche Ordnung und Politik in Tansania, 1920-1970. München: Oldenbourg 2007, S. 157, 195 f.

41 Siehe z.B. Eric J. Hobsbawm: Nationen und Nationalismus. Mythos und Realität seit 1780. Frankfurt/M. u. a.: Campus 1992, S. 50. 
Literaten stellte sich mithin die Frage: Sollten sie für eine internationale Leserschaft schreiben oder für ein lokales Publikum, das zu großen Teilen noch kaum lesefähig war?

In der zweiten Hälfte der 1960er Jahre betrieb man die nationalistische Sprachpolitik mit Nachdruck. Verwaltung und Dichtkunst bildeten auch in diesem Fall einen wirksamen Verbund. Allmählich entstand eine Swahili-Literatur, die langfristig die „Swahilization of Tanzanian culture“42 beförderte. Im Juni 1968 versammelte der Staatspräsident eine Gruppe von Schriftstellern im hauptstädtischen Regierungssitz und forderte sie auf, „to use their talents in order to promote a better understanding by the people of the land [...] of national politics“. ${ }^{43}$ In der Folge gründete sich eine Society for Swahili Composition and Poetry in Tanzania, die nicht nur die Förderung der Swahili-Literatur betrieb, sondern auch die politische Bildung im Sinne der Staatsführung. Zunächst aber musste sich die tansanische Nationalsprache gegen die Sprache der Kolonialherren durchsetzen. 1962 hatte in Kampala, der Hauptstadt Ugandas, eine Konferenz afrikanischer Autoren, darunter Chinua Achebe, Wole Soyinka, John Pepper Clark und Ezekiel Mphahlele, stattgefunden, die zunächst die Entstehung eines englischsprachigen Romans in Afrika vorbereitete. ${ }^{44}$ Nach Peter Palangyos Dying in the Sun aus dem Jahr 1967 war Village in Uhuru der zweite von einem tansanischen Autor in englischer Sprache verfasste Roman überhaupt. ${ }^{45}$

Als Ruhumbika an diesem Text arbeitete, verfolgte die tansanische Regierung eine sozialistisch ausgerichtete Entwicklungspolitik. Schon 1962 war Nyereres Schrift Ujamaa - The Basis of African Socialism erschienen. ${ }^{46}$ „Ujamaa“ ist Swahili für ,Familiensinn“ oder ,Gemeinschaftssinn‘. Politisch wirksam wurde diese Konzeption nach der Arusha-Deklaration vom Februar 1967. Dieses Datum markiert einen Bruch mit aus der Kolonialzeit stammenden und durch die Weltbank bekräftigten Entwicklungsdirektiven. Nyerere erklärte einen ländlichen Sozialismus zum offiziellen Ziel der Regierungspolitik und unterstrich die Not-

42 Mazrui: Swahili Beyond the Boundaries (Anm. 33), S. $28 \mathrm{f}$.

43 Lyndon Harries: Poetry and Politics in Tanzania. In: Ba Shiru 4 (1972), S. 52-54, hier S. 52.

44 Vgl. Elena Bertoncini-Zúbková: Outline of Swahili Literature. Prose, Fiction and Drama. Leiden: Brill ${ }^{2} 2000$, S. 74.

45 Später hat Ruhumbika wie die meisten Schriftsteller Tansanias in der Nationalsprache geschrieben und sich für die Förderung der Swahili-Literatur auch als Universitätslehrer eingesetzt. Vgl. Bertoncini-Zúbková: Outline of Swahili Literature (Anm. 44), S. 86.

46 Julius K. Nyerere: Ujamaa - The Basis of African Socialism. In: J.K.N.: Freedom and Unity (Anm. 23), S. 162-171. Nyerere hatte schon zuvor sozialistische Ideen artikuliert, diese hatten bis 1967 jedoch kaum Einfluss auf die Politik der Regierung. Siehe dazu Cranford Pratt: The Critical Phase in Tanzania, 1945-1968. Nyerere and the Emergence of a Socialist Strategy. Cambridge: Cambridge University Press 1976. 
wendigkeit umfassender Umsiedlungen. Bis 1985 hielt man an diesem Kurs fest. Noch heute ist die sozialistische Phase Tansanias ein Thema literarischer Erzählungen. Neben der sozialistisch engagierten hatte sich bald auch eine kritische Ujamaa-Literatur herausgebildet. ${ }^{47}$ Ruhumbika hat 1992 in Dar es Salaam den in Swahili geschriebenen Roman Miradi Bubu ya Wazalendo (,Die unsichtbaren Unternehmungen der Patrioten'), erscheinen lassen, dessen Handlung bis in die 1980er Jahre reicht. ${ }^{48}$ Gleichsam die Vorgeschichte dazu hatte der ebenfalls aus Ukerewe gebürtige Aniceti Kitereza 1945 in Kerewe geschrieben. Ruhumbika bevorwortete und übersetzte das Familienepos 2002 unter dem Titel Mr. Myombekere and His Wife Bugonoka. Their Son Ntulanalwo and Daughter Bulihwali ins Englische. ${ }^{49}$ Sein erster Roman, fertiggestellt nach der Arusha-Deklaration - die Vorrede an den Leser ist auf Juni 1968 datiert - handelt dagegen vom Aufbruch des Dorfes und damit der gesamten Nation in eine Zukunft, die bereits im Zeichen des Sozialismus steht:

Nyerere was right. The country was poor but infinitely rich, because it had a united people. The settlement in Wantu was for Chamambo men, but in the spirit of ujamaa what concerned Chamambo concerned the rest of Wantu no less, since it concerned the whole of Tanzania. (VU, S. 112)

Wie schon die Schwarzwälder Dorfgeschichten so ist auch Village in Uhuru den thematisierten Veränderungsprozessen nicht äußerlich. In beiden Fällen stellt sich mithin die Frage nach der gesellschaftlichen Funktion der Literatur. Stärker als es im Rahmen des vorliegenden Aufsatzes möglich ist, wären in dieser Hinsicht jene sowjetischen Romane der 1920er und 30er Jahre zu berücksichtigen, die ebenfalls von einer Veränderung ländlicher Lebensformen handeln, welche sich hier allerdings sehr viel deutlicher als Kollektivierung darstellt. Das gilt für Platonovs Čevengur (,Tschewengur', ca. 1929), Šolochovs Podnjataja celina (,Neuland unterm Pflug‘, Bd. 1, 1932) oder Fedor Panferovs Bruski (,Wolgabauern', 1937) wie auch für die chinesischen Romane des Šolochov-Übersetzers und Stalinpreisträgers Zhou Libo Baofeng zhouyu (,Orkan“ 1948) (50 $^{\text {und }}$

47 Beispiele für sozialistisch inspirierte Ujamaa-Literatur sind Kulikoyela K. Kahigi / R.A. Ngerema: Mwanzo Wa Tufani („Der Beginn eines Sturms“,1976) und John Ngomoi: Ndoto Ya Ndaria („Ndarias Traum“, 1976). Einer kritischen Ujamaa-Literatur werden zugerechnet: Ngalimecha Ngahyoma: Kijiji Chetu („Unser Dorf“, 1975), George Liwenga: Nyota ya Huzuni („Der Stern der Bekümmernis“, 1980) und Euphrase Kezilahabi: Dunia uwanja wa fujo („Die Welt ist ein Ort des Chaos“, 1975).

48 Siehe dazu Jan Blommaert: The Sociolinguistics of Globalization. Cambridge: Cambridge University Press 2010, S. 64-78.

49 Siehe dazu Geider: Die Ökumene (Anm. 34), S. 383-392. 
Shanxiang jubian (,Großer Wandel in einem Bergdorf‘, Bd. 1, 1958), Ding Lings Taiyang zhao zai Sanggan he shang (,Sonne über dem Sanggan', 1948) oder Liu Qings Chuangye shi (,Die Erbauer', 1960). ${ }^{51}$ Von einer gewissen Präsenz des sozialistischen Realismus in Tansania ist jedenfalls auszugehen. ${ }^{52}$

\section{2}

Ort der Handlung von Village in Uhuru ist das auf einer von den Wantu bewohnten Insel im Lake Mamamaji gelegene Dorf Chamambo. Dort befindet sich auch die Kleinstadt Chanzio. Im Unterschied zur Hauptstadt Dar es Salaam, einem weiteren Schauplatz, sind diese Orte wohl fiktiv, zumindest aber fiktionalisiert. In verschiedener Hinsicht erinnern sie immerhin an die Insel Ukerewe. „Mamamaji“ ist Swahili und bedeutet ,Mutter der Gewässer', „Wantu“ ist eine Abwandlung von ,Watu' und bedeutet ,Menschen'. „Chamambo“ heißt ein Dorf ,mit Angelegenheiten“; das dem Roman angehängte Glossar übersetzt „mambo“ ins Englische mit „matters“ (VU, S. 200). Damit ist dieser Geschichte der Name der Gattung gewissermaßen eingeschrieben. Chamambo ist ein exemplarisches Dorf und erzählt wird von Dingen und Angelegenheiten, die für seine Bewohner von Belang sind. Doch verweist der Dorfname nicht allein auf eine literarische Form, in der sich dieses Erzählen vollzieht; er verweist zunächst auf Geschichten, die im Dorf selbst zirkulieren, auf eine Form des alltäglichen Erzählens also und damit auf ,Dorfgeschichten' in einem älteren Sinn des deutschen Worts. Bevor nämlich Anfang der 1840er Jahre erstmals eine literarische Erzählform so genannt wurde, bedeutete ,Dorfgeschichte', das ist anhand von Belegen aus dem 18. Jahrhundert rekonstruiert worden, „eine im Dorf umlaufende mündliche Erzählung“"53 - auch in diesem Sinn ist Chamambo ein Dorf mit Geschichten. Zu den Schauplätzen zählt weiterhin „new Chamambo“ (VU, S. 114). Ein zentrales Handlungselement ist die Errichtung einer geschlossenen Siedlung auf einem vormaligen Missionsgelände: „1,250 new houses with green-painted roofs stood on the Kilima slope

50 Siehe dazu Richard King: Milestones on a Golden Road: Writing for Chinese Socialism, 1945-80. Vancouver: University of British Columbia Press 2013, S. 46-69.

51 Siehe dazu Joe C. Huang: Heroes and Villains in Communist China. The Contemporary Chinese Novel as a Reflection of Life. London: C. Hurst \& Company 1973.

52 Nach der Arusha-Deklaration war u. a. Maxim Gorkis Matj (,Die Mutter', 1906/07) in Swahili erschienen; vgl. Mazrui: Swahili Beyond the Boundaries (Anm. 33), S. 147.

53 Wolfgang Seidenspinner: Spuren alltäglichen Erzählens im 18. und 19. Jahrhundert. „Dorfgeschichten“ als sozial definierte, offene Form von Kommunikationsprozessen. In: Fabula 35 (1994), S. 63-80, hier S. 71. 
overlooking the lake“ (VU, S. 112). Das alte ,Dorf“ wird einer traditionellen, während des Kolonialzeitalters kaum veränderten Siedlungsweise ${ }^{54}$ entsprechend als bloße Ansammlung einzelner Gehöfte beschrieben: „The Wantus did not live in communities. Each man occupied his own corner with his people.“(VU, S. 10) Die im Übergang vom alten zum neuen Chamambo, im Zuge der Dorfbildung also, aufbrechenden Konflikte sind das eigentliche, in der zweiten Romanhälfte entfaltete Thema der Erzählung.

Ausgetragen werden die Konflikte von den Haupthandlungsträgern, das sind: Musilanga, Oberhaupt des Dorfes, und Balinde, sein Sohn. Letzterer ist der eigentliche Protagonist dieser Geschichte, und vorwiegend aus seiner Sicht wird das Geschehen dargestellt. Von Balindes Jugend handelt die erste Hälfte des Romans, der auch ein ,Entwicklungsroman“ ist, und zwar, so möchte man hinzufügen, in einem ungewöhnlichen Doppelsinn: Die Persönlichkeit des Helden entwickelt sich in einem durch sein eigenes Handeln mit verursachten Konflikt, insofern er die ,Entwicklung“ („development“, S. 114 u.ö.) des Landes aktiv betreibt. Der Rückgriff auf das in der Literatur des Sozrealismus prominente Motiv des Vater-Sohn-Konflikts ${ }^{55}$ erlaubt es Ruhumbika, mit der Geschichte von Musilanga und Balinde auch die einer Dorfgemeinschaft und darüber hinaus die Geschichte der tansanischen Nation zu erzählen.

Dem Wunsch seines Vaters gemäß tritt der Sohn im Januar 1947 in das koloniale „Tanganyika Territory education system“ (VU, S. 16) ein; später wählt er die Laufbahn zum Lehrerberuf. Das Cambridge School Certificate soll den Besuch des Makerere College in Uganda ermöglicht: eine für die Bildungselite des kolonialen Tanganyika typische Laufbahn, wie sie auch Nyerere und Ruhumbika durchlaufen haben. Um auf einer Missionsschule die englische Sprache der Kolonialherren erlernen $\mathrm{zu}$ können, muss Balinde sich taufen lassen und $\mathrm{zu}$ „Benjamin Balinde Musilanga“ (VU, S. 39) werden. Wegen einer Liebesaffäre von der Schule verwiesen, kehrt er zunächst nach Chamambo zurück. Sich den Vorstellungen des Vaters gemäß im Dorf anzusiedeln, „like ordinary people who had not gone to school“" (VU, S. 51) das Land zu bebauen und auf Fischfang zu gehen, kommt für Balinde nicht in Frage. Er bricht auf nach Dar es Salaam, um Lohn-

54 Siehe zu den unterschiedlichen traditionellen Siedlungsformen John E. Moore: Traditional rural settlement. In: L. Berry (Hg.): Tanzania in Maps. London: University of London Press 1971, S. 124-127. Eine konzise Darstellung der Siedlungsgeschichte bietet Idris S. Kikula: Policy Implications on Environment: The Case of Villagisation in Tanzania. Uppsala: The Nordic Africa Institute 1997, S. 17-23.

55 Siehe dazu Katarina Clark: Socialist Realism with Shores: The Conventions for the Positive Hero. In: Thomas Lahusen / Evgeni Dobrenko (Hg.): Socialist Realism without Shores. Durham: Duke University Press 1997, S. 27-50. 
arbeit zu suchen. Schon auf der Eisenbahnreise durch das Land, erfährt er von Nyereres Partei. Bald angesteckt vom „fire of nationalism“ (VU, S. 66), tritt er der TANU Youth League bei, dem militanten Kern der tansanischen Einheitspartei. Als im Sommer 1958 Parteibüros im ganzen Land und so auch in Wantu eröffnet werden sollen, geht Balinde als Assistent des Parteifunktionärs Zidori Kiboga nach Chanzio, um die ländliche Bevölkerung für den Aufbau der Nation zu mobilisieren. „We are essentially an agricultural country, and it is on the farmers, on the man in the village, that we will build our country“ (VU, S. 69), lautet die Parole. Balinde kehrt also nach Wantu zurück, in der irrigen Gewissheit, sein Vater werde stolz auf ihn sein.

Musilanga wird als ein traditional legitimierter Dorfvorsteher eingeführt, der diese Stellung von seinem Vater übernommen hat. Mit seiner Familie bewohnt er eine Hofanlage, in deren Mitte ein alter Baum steht, von einem Ahnen gepflanzt, der bei der Gründung Chamambos als Vorsteher eingesetzt wurde. Von den Bewohnern wie auch von den Angehörigen seines Hauses wird Musilanga als Autorität und als „a proper Wantu“ (VU, S. 3) geachtet. In jeder Hinsicht steht der Alte für einen konservativen Traditionalismus und für ein Tansania der Stammesverbände, gegen das die Idee der Nation durchgesetzt werden muss: „To him country meant only one thing: Wantu“ (VU, S. 175). Nach der Unabhängigkeit hofft er in Erinnerung einer „golden past“ (VU, S. 84), der Patriarch der Insel werde zurückkehren und „really rule his country like his grandfather, Makaka, before the coming of the Germans“ (VU, S. 83). Vor allem aber stemmt der Alte sich gegen die Dorfbildung. Sein Gehöft ist ihm ein geweihter Ort, an dem seine Vorfahren begraben liegen (VU, S. 182). Es aufzugeben und die Stätte der landwirtschaftlichen Nutzung zu überlassen, betrachtet er als ein Sakrileg. Darum verweigert Musilanga die Umsiedlung in das für ihn vorgesehene Haus in Neu-Chamambo: „How could Musilanga see that settlement as progress? To him it was the worst of prisons." (VU, S. 181) Die Sichtweisen könnten unterschiedlicher nicht sein: Die Proponenten des Wandels sehen „that settlement as the beginning of the flow of water which had from time immemorial been stagnant, in the right direction. Musilanga saw it as the final violation of their society“ (VU, S. 180). Auch diese Sicht vermittelt der Erzähler, indem er mitunter die Perspektive des Alten einnimmt. Dessen Gedanken, geister- und hexengläubige Vorstellungen einschließlich (vgl. VU, S. 182), werden öfters in erlebter Rede wiedergegeben, um die Ablehnung der Modernisierung für den Leser nachvollziehbar zu machen. Auch Balinde gehört in den Augen seines Vaters am Ende zu jenen, die das Land der Wantu vernichtet haben, „killed their country, that is, destroyed society such as he conceived it. Society in which his father and his forefathers had lived. The Wantu in which he was born and its life as he had been living it.“(VU, S. 174) 
Der zentrale Konflikt dieser Erzählung ist keine beliebige Anleihe bei der literarischen Tradition. Die besondere Bedeutung des Vater-Sohn-Konflikts verdankt sich einer gesellschaftlichen Dynamik des Wandels, die in der afrikanischen wie in anderen Literaturen dieses Motiv hervortreibt. Es setzt die Lebenszeit von Individuen in Bezug zur historischen Zeit von Gemeinschaften und macht die Unterschiede der lebensweltlichen Lagerung sichtbar. Spannungen, die wohl immer schon im Verhältnis zwischen den Generationen auftraten, erfahren in einer dynamischen Zivilisation, wie Village in Uhuru sie darstellt, eine Verschärfung: Die Alten büßen ihre in gesammelter Erfahrung begründete Weisungskompetenz ein. Mit zunehmender Distanz zwischen Erfahrungsraum und Erwartungshorizont verliert solche Weisheit an Wert; damit schwindet die Autorität ihrer Träger. Die Wertlosigkeit des Erfahrungswissens kehrt Ruhumbika hervor. So lässt er die „old ones“ (VU, S. 77) die nach nationaler Unabhängigkeit strebenden Jungen davor warnen, die Europäer aus dem Land zu vertreiben, wie es angeblich in Ghana geschehen sei:

„My child,“ he began speaking through the nose to keep the snuff in position, „you are young and you have travelled much more than we your fathers. [...] Have you ever heard of a black man who knows how to make even a needle? The clothes we wear, the money for which we labour, and for which your Nyerere is cheating those people, belong to the Mzungu. A liar catches himself. Where will your Nyerere get those things which he says will make people rich when the Mzungo goes away?“ Nobody there could help pitying the ignorance of the youth. (VU, S. 77)

Die Erzählung lässt indes keinen Zweifel daran, dass die Alten die eigentlich Ignoranten sind. Die Jugend, die Youth League der Partei allen voran, hat die Zukunft des Landes längst in ihre Hände genommen und es ist überwiegend die Perspektive des jungen Balinde, mit der Ruhumbika die gesellschaftlichen Veränderungen darstellt. Der Erzähler steht dem Konflikt zwischen Vater und Sohn nicht neutral gegenüber; seine Parteinahme ist mit einer Bewertung des Geschehens verbunden. Dem Leser wird die Bejahung der gesellschaftlichen Transformation nahegelegt, für die Balinde sich einsetzt: Nationbuilding und Development.

Diese Deutungskategorien müssen an den Text nicht herangetragen werden. Er entstand in und er handelt von einer Zeit, in der diese historischen Konzepte für das Handeln der maßgeblichen Akteure leitend waren. Viele der in die Unabhängigkeit entlassenen Länder Afrikas erbten von den Kolonialherren einen zentralisierten Verwaltungsapparat, den sie zunächst auf solche Entwicklungsziele hin ausrichteten, die ebenfalls im letzten Jahrzehnt des Kolonialismus noch vorgezeichnet worden waren. Noch in der Endphase der britischen Herrschaft waren auch Umsiedlungsmaßnahmen ins Auge gefasst worden. 1960 hatten 
Berater der World Bank Mission ein erstes Gutachten über die wirtschaftliche Lage des damaligen Tanganyika erstellt und entwicklungspolitische Empfehlungen ausgesprochen. ${ }^{56}$ Den Plänen der britischen Kolonialverwaltung entsprechend konzentrierte man sich zunächst auf die Landwirtschaft. Deren Verbesserung sollte die Erzeugung von überschüssigen Handelsgütern ermöglichen und damit das Kapital für eine spätere Industrialisierung erbringen. Neben dem auf eine kurzfristige Produktionssteigerung durch kleinere Veränderungsmaßnahmen zielenden Improvement Approach wurde ein Transformation Approach angeraten, der eine schnelle Modernisierung durch Einsatz kapitalintensiver Techniken im Rahmen von „planned farms of economic size“ ${ }^{\text {"57 }}$ zum Ziel hatte. Es galt, die verfügbaren Ressourcen in einigen ausgewählten Gebieten mit guten Anbaubedingungen zu konzentrieren. Dabei kam die zerstreute Siedlungsweise als Entwicklungshemmnis in den Blick. Aus Sicht der Planer war eine Umsiedlung erforderlich. Sie würde die ländliche Bevölkerung auch aus überkommenen soziokulturellen Bindungen herauslösen. Auf diesem Wege wollte man konservative Bauern zur Übernahme neuer Regeln und Praktiken bringen. ${ }^{58}$ Die Studie der Weltbank wurde später zur Grundlage der ersten Entwicklungspläne, die das unabhängige Tansania für die Jahre 1961-64 und 1964-69 aufstellte..$^{59}$ Allerdings setzte man nicht länger auf große landwirtschaftliche Betriebe. Zentraler Bestandteil der Pläne war ein Village Settlement Scheme.

Die Romanerzählung nimmt auf diese Zusammenhänge direkt Bezug. Von „Transformation Approach“ (VU, S. 110), „collective villages approach“ (VU, S. 110) und „Five Years Development Plan“ (VU, S. 108) ist ausdrücklich die Rede. Namentlich mehrfach genannt wird im Roman Julius Nyerere. Dessen Parlamentsrede, in der er am 12. Mai 1964 den ersten Fünfjahresplan vorstellte, findet der Leser mit präziser Datumsangabe ausführlich zitiert. Die im Roman angegebene Zahl von 60 Pilotdörfern entspricht ungefähr den historiographischen Angaben ${ }^{60}$ wie auch die Absichtserklärung mit den offiziellen Verlautbarungen tansanischer Politiker dieser Zeit übereinstimmt: „Communal villages would be founded to enable the people to use their united efforts to the maximum and to facilitate the

56 International Bank for Reconstruction and Development (IBRD): The Economic Development of Tanganyika. Baltimore: Johns Hopkins Press 1961; The Tanganyikan Five Year Plan for Social and Economic Development July 1964-June 1969. Dar es Salaam: Government Printer 1964.

57 IBRD: The Economic Development of Tanganyika (Anm. 56), S. 6.

58 Vgl. IBRD: The Economic Development of Tanganyika (Anm. 56), S. 131.

59 Vgl. Michael Jennings: Surrogates of the State. NGOs, Development, and Ujamaa in Tanzania. Bloomfield: Kumarian 2008, S. 38-42.

60 Der erste Fünfjahresplan sah 69 Dörfer vor. Vgl. Jennings: Surrogates of the State (Anm. 59), S. 42. 
Government's work in improving their agricultural methods and in raising their standard of living.“ (VU, S. 108) Als Voraussetzung einer modernen Landwirtschaft hatte Nyerere die Dorfbildung bereits in seiner Antrittsrede propagiert: „The first and absolutely essential thing to do [...], if we want to be able to start using tractors for cultivation, is to begin living in proper villages. "61 Unter dem Titel Socialism and rural development ${ }^{62}$ verbindet er 1967 dann die Idee der Dorfbildung mit dem Konzept der ,Ujamaa‘ zur Leitvorstellung einer „nation of ujamaa villages where the people co-operate directly in small groups and where these small groups co-operate together for joint enterprises".${ }^{63}$ Sowohl in Israel als auch in China hatte sich Nyerere bei Staatsbesuchen über Formen der ländlichen Kollektivierung informiert. Dem Landwirtschaftsminister von Tanganyika war bei einem Israelbesuch die Unterstützung von Agrarexperten bei der Ausbildung von Farmern und der Gründung von Siedlungen nach dem Vorbild der Kibbutzim und Moshawim versprochen worden. Im Rahmen der Unabhängigkeitsfeier hatte Israel Stipendien für Studienaufenthalte zugesagt. ${ }^{64}$ Und so befindet sich auch Balinde, als sein Land die Unabhängigkeit erhält, „with fifty-nine compatriots in a kibbutz in Israel“ (VU, S. 87), wo er sechs Monate lang am Leben eines ländlichen Kollektivs teilnimmt.

Mit der Forderung nach „proper villages“ war eine bestimmte Konzeption des Politischen verbunden. Nicht nur über Fragen der wirtschaftlichen Organisation, auch über Regierungsformen wurde im Tansania der 1960er Jahre intensiv diskutiert. In einer Monarchie, so heißt es in einem Positionspapier der TANU-Partei von 1966, sei der König keine Person, er sei vielmehr gleichwertig einen kleinen Gott; im Rahmen einer Parlamentsdebatte wurde auch an die britischen Königsmorde erinnert. ${ }^{65}$ Befürwortet wurde dagegen eine bestimmte Form der Volksherrschaft, direkte Demokratie. Nyerere berief sich auf die griechische Antike:

The ancient Greeks lived in small towns. Each town was a complete ,nation“ with its own government. They did not have kings or watemi [Chiefs; M.T.]. Their governmental affairs were considered and decided in a meeting of all commoners together. Authority and

61 Nyerere: President's Inaugural Address (Anm. 23), S. 184.

62 Julius K. Nyerere: Socialism and rural development. In: J.K.N.: Freedom and Socialism. Uhuru na Ujamaa. Oxford: Oxford University Press 1968, S. 337-366.

63 Nyerere: Socialism and rural development (Anm. 62), S. 365.

64 Vgl. Steven Carol: From Jerusalem to the Lion of Judah and Beyond: Israel's Foreign Policy. Bloomington: iUniverse 2012, S. 75; siehe auch Abel Jacob: Foreign Aid in Agriculture: Introducing Israel's Land Settlement Scheme to Tanzania. In: African Affairs 71 (1972), S. 186-194.

$65 \mathrm{Vgl}$. Steven Feierman: Peasant Intellectuals: Anthropology and History in Tanzania. Madison: University of Wisconsin Press 1990, S. 228 f. 
responsibility did not rest with a single individual or a small group of citizens, but rested with the entire citizenry together. The Greeks called this governmental arrangement ,Demokratia,' that is, government by the citizens, in order to distinguish it from royal government and others. ${ }^{66}$

Die Dorfbildung sollte die Entstehung einer direkten Demokratie in Tansania befördern. Denn erst eine derart verdichtete Siedlungsweise würde politische Versammlungen und Deliberation auf lokaler Ebene ermöglichen: „Living in villages will lead to true democracy in the matters which concern each village. “67

Offenkundig ist das hier begegnende Dorfkonzept von Zügen der Idealisierung geprägt, die sich schon für die deutschsprachige Diskussion des 19. Jahrhunderts nachweisen lassen. ${ }^{68} \mathrm{Im}$ vorliegenden Zusammenhang findet sich das ökonomische Ideal einer ,kommunistischen' Produktion auf der Grundlage von Gemeinbesitz mit dem politischen Ideal unmittelbarer Volksherrschaft verbunden. Diese Gemeinsamkeit wäre nicht etwa hinsichtlich eines geteilten Bezugs auf antike Quellen zu erklären. Vielmehr wären verflechtungsgeschichtlich, was im Rahmen des vorliegenden Aufsatzes nicht möglich ist, die Transfers und Aneignungen eines wandelbaren Dorfkonzepts nachzuzeichnen, das aus der Rechtsgeschichte seinen Weg in die sich formierende Anthropologie, in die koloniale Verwaltungspraxis, aber auch zu Anarchisten, Sozialrevolutionären und Theoretikern des Kommunismus, in die sozrealistische Literatur und weiter zu antikolonialen Widerstandsbewegungen und postkolonialen Modernisierern gefunden hat. Für die Geschichte von Tansania blieb diese Vision einer sozialistischen Gesellschaft, die sich in Dörfern demokratisch organisiert, bis in die 1980er Jahre von Bedeutung. Nach dem Scheitern seiner Entwicklungspolitik trat Nyerere 1985 freiwillig zurück, sein Nachfolger Ali Hassan Mwinyi leitete in enger Abstimmung mit dem Internationalen Währungsfond und der Weltbank den Übergang zu einer marktwirtschaftlich organisierten Gesellschaft ein.

In der wissenschaftlichen Literatur wird vorwiegend die zweite, mit der Arusha-Deklaration beginnende Phase der Villagization behandelt. Zum Zeit-

66 Julius K. Nyerere: Misingi ya Demokrasi (1966), zit. n. der englischen Übersetzung in Feierman: Peasant Intellectuals (Anm. 65), S. 226.

67 Julius K. Nyerere: Hotuba ya Rais (1962), zit. n. der englischen Übersetzung in Feierman: Peasant Intellectuals (Anm. 65), S. 228.

68 Siehe dazu Marcus Twellmann: „Ueberbleibsel der ältern Verfassung“ - Zur primitivistischen Imagination des Dorfes im 19. Jahrhundert. In: Werner Nell / Marc Weiland (Hg.): Imaginäre Dörfer. Zur Wiederkehr des Dörflichen in Literatur, Film und Lebenswelt. Bielefeld: Transcript 2014, S. 227-248. 
punkt der Deklaration gab es nicht mehr als 48 registrierte Dörfer; 95\% der Landbevölkerung lebte in verstreut liegenden Gehöften. ${ }^{69}$ Bis Dezember 1968 wurden 180, bis Dezember 1969650 Dörfer errichtet. Im Rahmen des zweiten Fünfjahresplans (1969-74) wurden die Umsiedlungsmaßnahmen durch eine zunehmend zentralisierte und ständig wachsende staatliche Verwaltung - zwischen 1967 und 1980 vervierfachte sich die Zahl der Verwaltungsposten ${ }^{70}$ - mit Nachdruck durchgesetzt. Schon 1968 hatte man auch Zwangsmaßnahmen angewandt, 1969 wurden diese durch ein Zirkular offiziell legitimiert. ${ }^{71}$ Im Rahmen der Operation Rufiji wurden die Bewohner eines ganzen, von Überflutungen bedrohten Flußtals in neu errichteten Dörfern angesiedelt. ${ }^{72}$ Ab 1970 verfolgte man einen so genannten ,Frontal Approach'. In den Daily News, einer in Dar-es-Salaam erscheinenden Tageszeitung, verkündete Nyerere am 7. November 1973 auf der ersten Seite, TANU habe beschlossen, dass bis 1976 sämtliche Bauern in Dörfern leben müssten. ${ }^{73} \mathrm{Zu}$ diesem Zeitpunkt gab es bereits mehr als 5.000 registrierte, nun als ,Planned“ oder ,Development Village‘ bezeichnete Dörfer mit mehr als 2,5 Millionen Einwohnern. In den folgenden Jahren sollten 3.000 Dörfer hinzukommen; bis 1977 wurden weitere 10,5 Millionen Menschen umgesiedelt ${ }^{74}$ - mit in mehrfacher Hinsicht verheerenden Folgen. ${ }^{75}$

Ruhumbikas Erzählung handelt von der ersten Phase der Dorfbildung. Vorbild für Chamambo mag das 1964 auf der Insel Ukerewe errichtete Dorf Gallu gewesen sein, eines der ersten Pilotprojekte, dessen erhoffter Erfolg die Bauern überzeugen sollte, sich in Dörfern anzusiedeln. ${ }^{76}$ In dieser frühen Phase, das gilt es zu berücksichtigen, hatte die tansanische Landbevölkerung ein gewisses Maß an Kontrolle über den Entwicklungsprozess. Die Regierung hatte das Konzept der ,Selbsthilfe' in ihr Programm integriert, um mit minimalen Ressourcen eine Verbesserung der Lebensbedingungen zu erreichen. Bevor Ende der 1960er Jahre ein zunehmend autoritärer Staat seine Steuerungshoheit zu behaupten und den Entwicklungsprozess unter Kontrolle zu bringen versuchte, konnten sich auf lokaler

69 Vgl. Dean E. McHenry: Tanzania's Ujamaa Villages. The Implementation of a Rural Development Strategy. Berkeley: Institute of International Studies 1979, S. 116.

70 Vgl. John Iliffe: Geschichte Afrikas. München: Beck 1997, S. 342.

71 Vgl. Andrew Coulson: Agricultural Policies in Mainland Tanzania. In: Review of African Political Economy 10 (1977), S. 74-100, hier S. 89.

72 Vgl. Coulson: Agricultural Policies in Mainland Tanzania (Anm. 71), S. 91 f.

73 Vgl. Pratt: The Critical Phase in Tanzania (Anm. 46), S. 293.

74 Vgl. Jennings: Surrogates of the State (Anm. 59), S. 48-51.

75 Siehe dazu James Scott: Seeing Like a State: How Certain Schemes to Improve the Human Condition Have Failed. New Haven: Yale University Press 1998, S. 223-261.

$76 \mathrm{Vgl}$. Kelly M. Askew: Les villages tanzaniens ujamaa 40 ans plus tard: moralisation et commémoration du collectivisme. In: Anthropologie et Sociétés 32 (2008), S. 103-132, hier S. 112. 
Ebene verschiedenste ungeplante und informelle Aktivitäten entfalten, die weitgehend in der Hand der Bevölkerung lagen. ${ }^{77} \mathrm{Zu}$ berücksichtigen ist außerdem, dass das erste Village Settlement Scheme gescheitert und 1966 offiziell aufgegeben worden war: Bis 1965 waren nur 23 Siedlungsprojekte angelaufen, von denen nicht mehr als sieben unter der Leitung der zuständigen Village Settlement Agency standen. ${ }^{78}$

\section{3}

Ruhumbikas Erzählung vermittelt die in den ersten Jahren der Unabhängigkeit herrschende Aufbruchstimmung und reflektiert zugleich die Schwierigkeiten, an denen der Versuch einer gesellschaftlichen Neuordnung zu scheitern drohte. Um Wirklichkeitsnähe bemüht, veranschaulicht der Text die versuchte Implementation eines Entwicklungsprogramms und die daraus resultierenden Verwerfungen im dörflichen Raum. Dabei geben die geschichtlichen Zusammenhänge nicht bloß einen Hintergrund der Dorfgeschichte ab, vor dem ein vermeintlich ewiger Konflikt zwischen Vater und Sohn sich in austauschbarem Zeit- und Lokalkolorit abspielte. Eigentlicher Gegenstand der Erzählung ist vielmehr der gesellschaftliche Prozess, insofern er an bestimmten Orten von bestimmten Akteuren getragen wird und darum auch eine dörfliche und eine Familienangelegenheit ist, verbunden mit „mambo“, was auch ,eine Kette dramatischer Ereignisse“ bedeuten kann..$^{79}$ Die Konkretisierung in der personalen Konstellation macht Strukturveränderungen darstellbar nach den Regeln der Erzählgattung und bringt die Perspektive derer zur Geltung, die den Wandel als ,Fortschritt“ - „maendeleo“ in Swahili (vgl. VU, S. 200) - begreifen, ihn vorantreiben und zu gestalten versuchen, wie auch die Sicht derer, die ihn erleiden und sich mitunter auch widersetzlich verhalten. Von besonderer Bedeutung, das gilt auch für Auerbachs Erzählungen, sind dabei intermediäre Akteure, die zwischen lokalen und translokalen Ordnungen vermitteln. Dazu zählen zum einen solche, die Interessen und Ansprüche des Dorfs und seiner Bewohner nach außen, gegenüber dem württembergischen Staat etwa, vertreten, Gemeinderatsvorsteher zum Beispiel. Als Mittler agieren zum anderen Akteure, die im Dorf den Staat repräsentieren, Dorfschulzen etwa, wie weiterhin Lehrer und Pfarrer, die vielfach ortsfremd sind und den

77 Siehe dazu Michael Jennings: We Must Run While Others Walk: Popular Participation and Development Crisis in Tanzania, 1961-9. In: Journal of Modern African Studies 41 (2003), S. $163-187$.

78 Vgl. Jennings: Surrogates of the State (Anm. 59), 43.

79 Ich danke Hashim Rungwe (Tübingen) für diesen Hinweis. 
Einheimischen ortsübergreifende Wissens- und Glaubensordnungen näher bringen. Eric Wolf hat in seinen Arbeiten über Mexiko auf die besondere Bedeutung solcher ,broker‘ für Transformationsprozesse in komplexen Gesellschaften hingewiesen: „They stand guard over the crucial junctures or synapses of relationships which connect the local system to the larger whole“. ${ }^{80}$

Die zentrale Bedeutung derartiger Mittlerfiguren kann als ein weiteres Merkmal der Gattung gelten. Die Dorfgeschichte entspricht darin einer bestimmten gesellschaftshistorischen Konstellation: Von besonderer Bedeutung sind Akteure dieser Art dort, wo die Regierungstätigkeit einer Zentralmacht nur eine geringe Durchdringungstiefe erreicht. Das ist für die ersten Jahrzehnte des Königreichs Württemberg ebenso festzustellen wie für die Kolonien und noch für die postkolonialen Staaten Afrikas. In dieser Situation ist die jeweilige Zentralmacht in hohem Maße angewiesen auf Intermediäre, die ihren Direktiven vor Ort zu praktischer Wirksamkeit verhelfen. Auch in Ruhumbikas Dorfgeschichte treten Vermittler beider Art auf. Da sind zum einen die Gegner des Entwicklungsprojekts wie Musilanga: Die gesellschaftlichen Einrichtungen des Kolonialzeitalters werden abgeschafft, ohne dass die vorkolonialen Verhältnisse - wie von den Traditionalisten erhofft - wiederhergestellt würden: „The Musilangas found themselves requested to accept another conception of ruling themselves" (VU, S. 87). Ruhumbika macht indes deutlich, dass jene „traditional monarchies“(VU, S. 88), die im Zuge der Nations- und Staatsbildung aufgehoben werden sollen, keineswegs in einer bruchlosen Kontinuität mit der ferneren Vergangenheit des Landes stehen. Die Deutschen hatten die ostafrikanischen Königstümer Ende des 19. Jahrhunderts zerstört, bevor die Briten Chiefs als Funktionäre der Kolonialverwaltung einsetzten. ${ }^{81}$ Da es den Kolonialherren nicht möglich war, mit eigenen Mitteln eine effektive Herrschaft über das Tanganyika Territory auszuüben, führte man 1925 das System der Indirect Rule ein. Lokale, ,traditionelle‘ Autoritäten sollten zwischen der afrikanischen Bevölkerung und der Kolonialverwaltung vermitteln. ${ }^{82} \mathrm{Zu}$ diesem Zweck wurden vorgefundene Gesellschaftsstrukturen manipuliert, wobei man sich auf die „mtemi, styled ,chief“ by the white man“ (VU, S. 2), wie es im Roman heißt, konzentrierte. Die Kolonialverwaltung war also auf Instanzen gestützt, die eine traditionale Form patrimonialer Herrschaft ausübten. Allerdings gab es im Zuge der „second colonial occupation“83 nach dem zweiten

80 Eric R. Wolf: Aspects of Group Relations in a Complex Society: Mexico. In: American Anthropologist LVII (1956), S. 1065-1078, hier S. 1075.

81 Vgl. Feierman: Peasant Intellectuals (Anm. 65), S. 8 f.

82 Siehe zur Stellung der Chiefs im System der Indirect Rule Iliffe: A modern history of Tanganyika (Anm. 29), S. 327 f. Siehe zur Entmachtung der Chiefs nach der Unabhängigkeit Feierman: Peasant Intellectuals (Anm. 65), S. 223-244. 
Weltkrieg Bestrebungen, in Tanganjika eine formal geregelte Verwaltung aufzubauen. Noch in der Kolonialzeit entstand somit eine Mischform patrimonialer und rational-bürokratischer Herrschaft, die vielfach als eigentliche Ursache prekärer Staatlichkeit in Afrika angesehen wird. ${ }^{84}$

Ruhumbika erzählt von einer Chief's Bill, die nach der Unabhängigkeit den Status der lokalen Autoritäten neu regelt: Erst die Engländer, das wird der Bevölkerung durch Gesandte des Ministeriums erläutert, hätten die Stammesführer zu bezahlten Dienern gemacht; von nun an blieben sie Führer nur solange, wie das Volk es wolle, „because our country is a democratic country, and in a democratic country it is the people who decide what they want“ (VU, S. 93). Aus demselben Grund sei niemand mehr verpflichtet, dem Stammesoberhaupt Geschenke darzubringen, damit er etwa für Regen sorge. Das hält den Royalisten Musilanga nicht davon ab, gelegentlich etwas zum Palast seines zumeist abwesenden Königs zu schicken, ${ }^{85}$ ist er es als Dorfvorsteher doch gewohnt, solche Gaben von anderer Seite entgegenzunehmen. Durch ein Dekret sind die klientelen Handlungs- und Beziehungsgeflechte patrimonialer Herrschaft offenbar nicht abzuschaffen. Den daraus resultierenden Schwierigkeiten für den Aufbau einer neuen Gesellschaft gilt Ruhumbikas Interesse.

In der geschilderten Phase der gesellschaftlichen Transformation werden die alten Mittler auf verschiedenen Ebenen durch neue ersetzt. Mitglieder der Einheitspartei treten als Area Commissioner an die Stelle der kolonialen District Commissioner, so auch Balinde, der aus Sicht seines Vaters damit zum Nachfolger des „white man“ wird (VU, S. 176). Parteimitglieder übernehmen darüber hinaus die lokale Verwaltung, indem sie an die Stelle der Dorfvorsteher treten. So wird Musilanga entmachtet und durch einen gewählten Vorsteher ersetzt, dessen Legitimität sich den neuen demokratischen Prinzipien gemäß dem Willen der Bevölkerung verdanken soll. An die Stelle des geachteten Alten tritt Swaleh Alli, ein junger Mann aus Chanzio. Als ein Fremder ist der neue Verwaltungschef zwar

83 D. Anthony Low und John Lonsdale: Introduction: Towards the New Order 1945-1963. In: D. A.L. / Alison Smith (Hg.): History of East Africa. Bd. 3. Oxford: Clarendon 1976, S. 1-63, hier S. 12, zit. n. Iliffe: A modern history of Tanganyika (Anm. 29), S. 436.

84 Siehe dazu Gero Erdmann: Apokalyptische Trias: Staatsversagen, Staatsverfall und Staatszerfall - strukturelle Probleme der Demokratie in Afrika. In: Petra Bendel / Aurel Croissant / Friedbert W. Rüb (Hg.): Demokratie und Staatlichkeit. Systemwechsel zwischen Staatsreform und Staatskollaps. Opladen: Leske und Budrich 2003, S. 267-292.

85 Siehe in diesem Zusammenhang Feierman: Peasant Intellectuals (Anm. 65), S. 5: „Chiefship itself had been abolished soon after independence. Nevertheless, it quickly became clear that peasants in many parts of the West Usambara mountains [...] were offering cows, labor, and quantities of food as tribute to chiefs who had inherited their positions from pre-colonial rulers. The tribute came most visibly at times when the rainy season was expected to start.“ 
keinem der Dorfbewohner zu Loyalität verpflichtet, doch versieht er sein Amt durchaus nicht im Sinne einer rationalen Bürokratie. Das hergebrachte Tributsystem ausnutzend nimmt Swaleh Alli Gaben der Bewohner entgegen und verkehrt vor allem mit den schönen Frauen des Dorfs. Eine Affäre mit einer der Frauen Musilangas führt zum Eklat. Der „new order of things“ (VU, S. 107) stellt sich mithin als ein wenig geordnetes Durcheinander von Altem und Neuem dar, in dem ,Korruption“ um sich greift - ein Thema auch der späteren, auf Swahili verfassten Erzählungen Ruhumibikas. ${ }^{86}$ Tatsächlich waren die Einparteiensysteme der postkolonialen Staaten im subsaharischen Afrika vom Klientelismus durchdrungen. ${ }^{87}$ Auch die Perspektive des ,korrupten' Funktionärs versucht der Roman indes zu vermitteln: „A jobless man, existing on an uncle hardly better off, a cobbler in that tiny town of Chanzio, could hardly help celebrating the heavensent luck of finding himself, overnight, headman of the whole village of Chamambo.“ (VU, S. 150)

Der Hauptperspektivträger jedoch ist Balinde. Musilangas Sohn, davon handelt die zweite Hälfte des Romans, wird mit der Umsetzung der Dorf- und Nationsbildungspläne beauftragt. Dieser einzige Sohn und vorbestimmte Nachfolger eines traditionellen Dorfoberhaupts kehrt also, durch die schulische Bildung und den Aufenthalt in der großen Stadt seiner Herkunftskultur entfremdet, als Repräsentant der Partei und „burning with the fire of nationalism“ (VU, S. 66) nach Wantu zurück. Wie die Kolonialherren so ist zunächst auch die Unabhängigkeitsbewegung angewiesen auf Agenten, die das Ansehen der lokalen Bevölkerung genießen und diese für die Sache der TANU-Partei zu gewinnen vermögen. Der selber als Intermediär agierende Chief der Wantu begreift diese Strategie sofort: „And so our politicians are such clever men. To convert the Wantu they have sent the famous son of Kiboga [...] and as his assistant the son of Musilanga“ (VU, S. 82). Nachdem die Unabhängigkeit errungen ist, soll Balinde auch weiterhin in seiner Heimat zwischen Staat und Bevölkerung vermitteln. Ruhumbika bezeichnet diese Vermittlungsarbeit präzise als eine der ,Übersetzung': „The difficult work of translating the intentions of new Tanganyika into actions in new Wantu was entrusted to Balinde.“(VU, S. 94) „Placing the affairs of the nation before his

86 Gabriel Ruhumbika: Uwike usiwike kutakucha. Arusha / Dar es Salaam: Eastern Africa Publications 1978; G.R.: Miradi Bubu ya wazalendo. Dar es Salaam: Tanzania Publishing House 2002. Siehe dazu Pierre-Marie Decoudras: A la recherche des logiques paysannes. Paris: Karthala 1997, S. $169 \mathrm{f}$.

87 Siehe dazu Nicolas van de Walle: Meet the new boss, same as the old boss? The evolution of political clientelism in Africa. In: Herbert Kitschelt / Steven Wilkinson (Hg.): Patrons, Clients, and Policies: Patterns of Democratic Accountability and Political Competition. Cambridge: Cambridge University Press 2007, S. 50-67. 
personal interests“ (VU, S. 147) treibt er die Implementation des staatlichen Entwicklungsprogramms ohne Rücksicht auf seine Familie voran. Er selbst wählt just Chamamabo für das Pilotprojekt aus, eben das Dorf seiner eigenen Herkunft, mit dessen Bewohnern er auch verwandtschaftlich noch immer verbunden ist. Die gesellschaftliche Neuordnung führt darum nicht nur zu Verwerfungen zwischen den Generationen, sie hat auch ein inneres Zerwürfnis zur Folge: „What did his efforts mean if his family hated what he was helping to create?" (VU, S. 117)

Diesem Zwiespalt gilt Ruhumbikas Hauptaugenmerk; mit erzählerischen Mitteln, mitunter auch die Haltung eines Soziologen einnehmend, arbeitet er ihn heraus: „In a community where family attachments reach their extremist extent“ (VU, S. 117) und „the love of father and son“ als „the very foundation of men's families“ (VU, S. 140) angesehen wird, zieht der Bruch mit den traditionellen Werten - „I am rather over-progressive“ (VU, S. 145) bemerkt Balinde an einer Stelle im Scherz - tiefe Verwerfungen nach sich. Das muss der Neuerer schmerzhaft erfahren, als ihm der Vater die Fürsprache bei der Anbahnung einer Heirat mit Hermelinda versagt. In dieser Angelegenheit bleibt die junge Generation angewiesen auf die Zustimmung der alten. Zumindest die Umworbene nimmt das Heiratsverbot ihrer Eltern hin, die den Bewerber aufgrund der im dörflichen Kommunikationsraum umlaufenden Gerüchte ablehnen. Hier also begegnen dem Leser ,Dorfgeschichten' im älteren Sinn: mündliche Erzählungen, die alltäglich unter den Bewohnern des Dorfs zirkulieren, kurz: „village gossip“ (VU, S. 161). Teils nimmt der Erzähler die Perspektive der nicht namentlich identifizierten „men and women" ein und gibt ihre Gedanken im Modus der erlebten Rede wieder (vgl. VU, S. 183 u.ö.). Teils spricht er aus größerer Distanz über sie: „Like any newsmongers, they were not interested in finding out the whys of things. All that interested them was diffusing what they noted and imagined." (VU, S. 117f.) Ausgehend von einem sehr lückenhaften Wissen und von „things heard“ (VU, S. 197) deuten die Leute Balindes Rolle im Prozess der gesellschaftlichen Veränderung wie auch seine Beziehung zum Vater auf unterschiedliche Weise; am Ende wird eine dieser Deutungen ,adopted as the truth“ (VU, S. 197): Balinde ist für sie einer, „who has denied all his relatives, including his parents“ (VU, S. 137). Dieser Auffassung sind auch die Nachbarn. Selbst die Verwandten Balindes meiden ihn und sehen bei unvermeidbaren Begegnungen in ihm nur noch den „Bwana Aryakamisona“ „Balinde, Linde, or son of Musilanga, completely disappeared.“ (VU, S. 129) Am Ende verliert er in den Augen der andern nach der Familien- auch die Stammeszugehörigkeit: „And so to his public Balinde stopped being a Wantu.“(VU, S. 149)

Den Lesern seines Romans macht Ruhumbika auf diese Weise vor allem solche Konflikte nachvollziehbar, die sich für die Protagonisten des Wandels aus der eigentümlichen Ambivalenz ihrer Zwischenstellung ergeben. Broker nehmen in dynamischen Gesellschaften eine Mittelstellung zwischen Altem und Neuem 
ein und verbinden unterschiedliche Gruppen. Dazu sind sie in der Lage, weil sie sowohl von der lokalen als auch von der nationalen Elite anerkannt werden. Auf der einen Seite verdankt ihre Legitimität sich verwandtschaftlichen Bindungen sowie einer gemeinsamen Sprache und geteilten Gewohnheiten, auf der anderen Seite verdankt sie sich einer höheren Bildung, der Migration in eine größere Stadt und der Teilhabe an einer stärker kosmopolitisch geprägten Kultur. Doch ist mit ihrer Zwischenstellung auch eine Gefährdung verbunden. Denn sie agieren im Spannungsfeld von lokalen Traditionen und translokalen Regelsystemen, und gehen dabei mehrfache, teils unvereinbare Bindungen ein.

Intermediäre Ambivalenz kennzeichnet nicht nur die Stellung der Hauptfigur dieser Erzählung, sondern auch den Erzähler, der, Balinde auf seinem Weg begleitend, das Geschehen dem Leser darstellt. Auch der Erzähler steht einerseits den Dorfbewohnern nahe und sucht ihre Sicht zu vermitteln, ist andererseits aber bestrebt, einen das ganze Land erfassenden Wandel mitsamt den daraus sich ergebenden Erfordernissen zu beschreiben. Die eingangs formulierte Arbeitsdefinition ist in dieser Hinsicht zu ergänzen: Die Dorfgeschichte stellt die Veränderung ländlicher Lebensformen im Zuge translokaler Prozesse aus lokaler Perspektive dar, einer Perspektive, das ist hinzuzufügen, die gekennzeichnet ist durch intermediäre Ambivalenz. In gewisser Hinsicht weist Auerbachs Poetologie der Dorfgeschichte darauf hin: Sie geht von der Annahme aus, „daß nur ein Eingeborner das Volksleben in seiner Innerlichkeit erfaßt“, ${ }^{88}$ nicht aber Fremde wie zugereiste Lehrer, Pfarrer oder Beamte. Eine Voraussetzung der volkstümlichen Literatur ist mithin, dass der Verfasser mit dem dörflichen Leben in hohem Maße vertraut ist und es mit einer Binnenperspektive zu sehen vermag. Doch befähigt diese Vertrautheit allein noch nicht zu seiner Darstellung. Die Innensicht muss stumm bleiben, wenn sie sich nicht mit einem Blick von außen verbindet. Erst eine Phase der „Entfremdung und Isolirung“ ermöglicht nach Auerbach Dichtung als „Heimkehr“; ${ }^{89}$ nur wer sich davon entfernt hat, „sieht das Heimische fast mit fremdem Auge, mit einem Blick, der auf Entferntem geruht und dem sich das Heimische neu erschließt““.90 Der schwäbische Dichter beschreibt mit dieser Möglichkeitsbedingung einer Ethnographie der eigenen Herkunftskultur seinen Lebensweg: Er musste Nordstetten verlassen und von Stadt zu Stadt mehrfach seinen Wohnsitz wechseln, um aus der Ferne von seinem Heimatdorf erzählen zu können. Einen ähnlichen Weg ist Gabriel Ruhumbika gegangen, der in ostafrikanischen Städten

88 Berthold Auerbach: Schrift und Volk. Grundzüge der volksthümlichen Literatur, angeschlossen an eine Charakteristik J.P. Hebel's [1846]. In: B.A.: Schriften zur Literatur. Hg. von Marcus Twellmann. Göttingen: Wallstein 2014, S. 7-173, hier S. 25.

89 Auerbach: Schrift und Volk (Anm. 88), S. 25.

90 Auerbach: Schrift und Volk (Anm. 88), S. 29. 
und in der französischen Hauptstadt gelebt hatte, als er seinen Roman schrieb. In beiden Fällen kann eine gewisse Ambivalenz der Erzählhaltung also mit der Lebensgeschichte des Verfassers in Zusammenhang gebracht werden.

Der Vergleich lässt über Ähnlichkeiten hinaus indes gewichtige Unterschiede hervortreten: Die Stellung zu den lokalen Zusammenhängen einerseits und zu translokalen Prozessen andererseits ist je besonders. Zwar hat Auerbach von solchen Prozessen Kenntnis, doch ist er darin deutlich weniger als Ruhumbika engagiert. Die Schwarzwälder Dorfgeschichten suchen nicht Verständnis für die Projekte der württembergischen Staatsverwaltung zu wecken, sie beschreiben vielmehr den Widerstand der ländlichen Bevölkerung und nehmen für diese Partei. Widersetzlichkeit kommt auch im tansanischen Roman zur Darstellung, doch wird sie hier vorwiegend aus der Sicht eines Verwaltungsangehörigen beschrieben. Ruhumbika hat einen Agenten der Modernisierung zum Hauptperspektivträger gemacht. Darin tritt der Unterschied zwischen Village in Uhuru und Auerbachs Geschichten wohl am deutlichsten zu Tage und daraus erhellt die Beschaffenheit dieser Erzählungen in verschiedenster Hinsicht. Während der Proponent einer ,volkstümlichen Literatur' Sitten und Gebräuche etwa vielfach beschreibt und in seinen Geschichten auch Volkslieder festhält, ist Ruhumbika an Folklore wenig interessiert. Jener stellt volkskulturelle Praktiken dar, die im Zuge der Etatisierung unterdrückt werden, dieser unterstützt mit literarischen Mitteln staatliche Entwicklungspläne und Bestrebungen zur Bildung einer kulturell einheitlichen Nation, die regionale und lokale Traditionen mitsamt den darin verankerten Identitäten in sich aufheben soll.

Village in Uhuru lässt somit einen Widerspruch, der für die Gattung konstitutiv ist, besonders deutlich hervortreten: Intermediäre Ambivalenz findet sich hier in einer Ausprägung, die fraglich werden lässt, ob über diese Dorfgeschichte noch gesagt werden kann, sie stelle die Veränderung ländlicher Lebensformen aus lokaler Perspektive dar - zu dominant scheint die Sichtweise eines Funktionärs translokaler Prozesse. Und doch ist auch in diesem Fall eine gewisse Ambivalenz gegeben. Im breiten Varianzspektrum der Gattung ist Ruhumbikas Erzählung nicht weit entfernt von jenen zum engeren Kanon des sozialistischen Realismus gehörigen Romanen, die ebenfalls von Dörfern handeln, die sich im Zuge von Kollektivierungsmaßnahmen verändern. Von einer ,ideologisch motivierten Eindeutigkeit der Wertung“", ${ }^{91}$ wie sie für Šolochovs Neuland unterm Pflug konstatiert wurde, kann hier jedoch nicht die Rede sein. Ruhumbika erzählt nicht von der

91 Hans Günther: Die Verstaatlichung der Literatur. Entstehung und Funktionsweise des sozialistisch-realistischen Kanons in der sowjetischen Literatur der 30er Jahre. Stuttgart: Metzler 1984, S. 94. 
gelingenden Bildung seines Protagonisten zu einem ,neuen Menschen', der in seinem Handeln über alle Hemmnisse hinweg und für andere vorbildlich die Idealvorstellung einer kommenden Gesellschaft verfolgt. ${ }^{92}$ Sein Held zeigt sich vielmehr tief verunsichert durch den Verlust seiner vormals im dörflichen Herkunftsraum verankerten Identität. Ob das Entwicklungsprogramm, dem er sich verschrieben hat, den erhofften ,Fortschritt' herbeiführen und das persönliche Opfer rechtfertigen wird, ist am Ende ungewiss. So erhellen aus dieser Geschichte vor allem gesellschaftliche Schwierigkeiten einer afrikanischen ,Modernisierung für Leser, die Ruhumbika - das lassen Sprache und Publikationsort vermuten vornehmlich außerhalb Tansanias gesucht hat. Im Roman ist von einem britischen Kolonialbeamten die Rede, der, vorinformiert durch ,all the ethnologists he had read up before coming to Africa“ (VU, S. 32), das sonderbare Handeln der Bewohner nur auf eine „African mentality“ (VU, S. 36) zurückzuführen weiß. Wer Village in Uhuru gelesen hat, weiß es besser.

92 Siehe zu diesem Muster der sowjetischen Romane Katerina Clark: Socialist Realism in Soviet Literature. In: Neil Cornwell (Hg.): The Routledge Companion to Russian Literature. London / New York: Routledge 2001, S. 174-183. 